\title{
(C) OPEN ACCESS \\ Secular trends and evaluation of complex interventions: the rising tide phenomenon
}

\author{
Yen-Fu Chen, ${ }^{1}$ Karla Hemming, ${ }^{2}$ Andrew J Stevens, ${ }^{2}$ Richard J Lilford ${ }^{1}$
}

\begin{abstract}
${ }^{1}$ Warwick Centre for Applied Health Research \& Delivery, Division of Health Sciences, Warwick Medical School, University of Warwick, Coventry, UK

${ }^{2}$ School of Health and Population Sciences, University of Birmingham, Birmingham, UK
\end{abstract}

\section{Correspondence to} Professor Richard Lilford, Warwick Centre for Applied Health Research \& Delivery, Division of Health Sciences, Warwick Medical School, University of Warwick, Coventry CV4 7AL, UK; R.J.Lilford@warwick.ac.uk

Received 7 May 2015 Revised 4 September 2015 Accepted 13 September 2015 Published Online First 6 October 2015

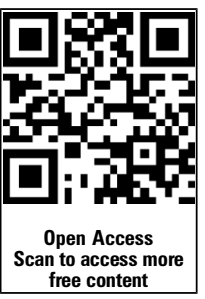

\section{SLinked}

http://dx.doi.org/10.1136/ bmjqs-2015-004842

\section{CrossMark}

To cite: Chen Y-F,
Hemming K, Stevens AJ, et al.
BMJ Qual Saf 2016;25:
303-310.

\begin{abstract}
Evaluations of service delivery interventions with contemporaneous controls often yield null results, even when the intervention appeared promising in advance. There can be many reasons for null results. In this paper we introduce the concept of a 'rising tide' phenomenon being a possible explanation of null results. We note that evaluations of service delivery interventions often occur when awareness of the problems they intend to address is already heightened, and pressure to tackle them is mounting throughout a health system. An evaluation may therefore take place in a setting where the system as a whole is improving - where there is a pronounced temporal trend or a 'rising tide causing all vessels to rise'. As a consequence, control sites in an intervention study will improve. This reduces the difference between intervention and control sites and predisposes the study to a null result, leading to the conclusion that the intervention has no effect. We discuss how a rising tide may be distinguished from other causes of improvement in both control and intervention groups, and give examples where the rising tide provides a convincing explanation of such a finding. We offer recommendations for interpretation of research findings where improvements in the intervention group are matched by improvements in the control group. Understanding the rising tide phenomenon is important for a more nuanced interpretation of null results arising in the context of system-wide improvement. Recognition that a rising tide may have predisposed to a null result in one health system cautions against generalising the result to another health system where strong secular trends are absent
\end{abstract}

\section{INTRODUCTION}

Interventions to combat health service delivery problems (such as hospitalacquired infections) are often developed in response to a heightened public awareness and mounting pressure to tackle them. Under these circumstances, a groundswell of public and professional opinion may be the stimulus for both a spontaneous change across a health system and formal evaluations of particular interventions within that system. Service delivery interventions are often complex in the sense that they are made up of a number of components, many of which may not be novel and which, unlike pharmaceuticals, are not restricted by licensing requirements. The result is that interventions of various types diffuse into widespread practice in an uncontrolled way while evaluation studies are under way. For example, concerns over hospital-acquired infections may lead hospitals across the system to adopt methods to improve hand hygiene and these same concerns may also stimulate formal research studies to evaluate specific interventions with the same aim. Insofar as these various interventions are effective, they produce a positive secular trend. We shall use the metaphor of a 'rising tide' as a short hand for such a secular trend that is contemporaneous with the evaluation of an intervention. Such a rising tide may obscure the measured effect of an intervention in a study with contemporaneous controls. Appreciating the possibility of a rising tide offers additional insight for interpreting null results where both control and intervention sites have improved.

This paper aims to illustrate the rising tide phenomenon in which this might explain a null result where both intervention and contemporaneous control sites have improved. We discuss evidence that may help distinguish between a rising tide and alternative explanations for the null result, and illustrate this approach with examples. 


\section{TEMPORAL TRENDS VERSUS OTHER EXPLANATIONS FOR IMPROVEMENT ACROSS INTERVENTION AND CONTROL SITES}

The possibility of a 'rising tide' explanation arises when a controlled study with baseline measurement(s) yields a null result in which there has been improvement across both intervention and control sites.

Various criteria can be put forward to help distinguish a rising tide from other explanations for such simultaneous improvement-we offer these in the spirit of Bradford Hill's famous criteria for causeeffect explanations in clinical research. ${ }^{1}$ Leaving aside the play of chance (which will have been calibrated statistically), the probability of the rising tide explanation increases in proportion to evidence for the existence of a rising tide and declines in proportion to evidence supporting rival explanations.

Evidence for a rising tide, from strongest to weakest, consists of the following:

1. Data showing that improvement similar to that in study sites occurred across the healthcare system as a whole. Such external data may be derived from regular population surveys, national registries, or routine administrative databases and provide direct evidence of a positive secular trend.

2. Data showing that the intervention and control sites within a study had started to improve before the intervention came on stream, so that it was a continuation of a trend in both intervention and control sites.
3. Qualitative evidence, say in the form of interviews with staff, showing strong motivation to improve practices in both intervention and control sites.

4. Circumstantial evidence in the form of press articles, government reports, and/or documents from national societies showing that the topic was one of pervading concern.

Contamination is the most immediate rival explanation for simultaneous improvement in both intervention and control groups. Contamination is used here in the standard epidemiological sense that control sites become aware of the intervention and replicate it to some degree, ${ }^{2}{ }^{3}$ thereby diluting the estimated effect; the direction of effect is from intervention sites to control sites within a study, biasing results towards the null. The intervention 'leaks' from intervention to control sites and must follow allocation to intervention and control conditions. A rising tide, by contrast, impacts on all sites in a system, irrespective of whether they are or are not included in the study and it may precede allocation of intervention and control groups. Contamination should be suspected when it can be demonstrated that participants in the control group were exposed to elements of the intervention that had 'spilled over' from the intervention group within the study (rather than from outside).

It is possible for other sources of bias (see table 1) to create or exaggerate the appearance of an improvement in the control group or even to create the

Table 1 Issues to be considered for assessing a rising tide phenomenon and results of assessment for the four case studies*

\begin{tabular}{|c|c|c|c|c|}
\hline & SPI2 & $\begin{array}{l}\text { Critical } \\
\text { pathways }\end{array}$ & EQHIV & MERIT \\
\hline \multicolumn{5}{|l|}{ Positive evidence } \\
\hline \multicolumn{5}{|l|}{ Direct evidence } \\
\hline $\begin{array}{l}\text { Improvement in process and/or outcome measures } \\
\text { observed in external sites: }\end{array}$ & Yes & Yes & Yes & Yes \\
\hline Timing: before or during evaluation study & $\begin{array}{l}\text { Before and } \\
\text { during }\end{array}$ & $\begin{array}{l}\text { Before and } \\
\text { during }\end{array}$ & Before & During \\
\hline System-wide or specific external site(s) & System-wide & $\begin{array}{l}\text { Specific external } \\
\text { sites }\end{array}$ & System-wide & $\begin{array}{l}\text { System-wide (but } 30 \% \\
\text { participation) }\end{array}$ \\
\hline $\begin{array}{l}\text { Qualitative evidence showing behaviour changes driven by } \\
\text { external factors in both study groups }\end{array}$ & Yes & Yes & Yes & Yes \\
\hline \multicolumn{5}{|l|}{ Suggestive evidence } \\
\hline $\begin{array}{l}\text { Baseline measures better than expected, or already } \\
\text { showing high standards or improving trend }\end{array}$ & Yes & Yes & Yes & Yes \\
\hline \multicolumn{5}{|l|}{ Circumstantial evidence } \\
\hline Heightened awareness of the problems & Yes & Yes & Yes & Yes \\
\hline \multicolumn{5}{|l|}{ Negative evidencet } \\
\hline Contamination within study & No & No & Unlikely & Unlikely \\
\hline Other potential sources of biases $\ddagger$ & Not apparent & Not apparent & $\begin{array}{l}\text { Attrition bias cannot be } \\
\text { ruled out }\end{array}$ & Not apparent \\
\hline
\end{tabular}


illusion of improvement in the intervention group, when in fact it was mainly or only the control group that had improved. Bias could arise, for example, if there was higher dropout from control than intervention sites or if controls were subject to selection bias.

\section{EXAMPLES OF A PUTATIVE RISING TIDE PHENOMENON}

In this section we provide four examples from published literature in which a rising tide phenomenon may be suspected. We briefly describe the key features of these studies and illustrate how the criteria mentioned above and listed in table 1 can be applied to help inform a judgement on the likelihood of a rising tide explanation versus alternative explanations.

Our first example, the Safer Patients Initiative phase 2 (SPI2) study, was a controlled before-and-after evaluation of a multicomponent hospital clinical safety programme. ${ }^{4} 5$ Many dimensions of quality measured in the study improved over the intervention period (spanning from March 2007 to September 2009), but did so equally in both intervention and control groups (figure 1). One of the targets of the intervention was to improve recognition of deteriorating patients in general wards, and the quality of nursing observations (as judged from masked review of the notes) improved markedly and statistically significantly over the study period, but no difference was observed in the rate of improvement across intervention and control sites. Likewise, use of hand washing materials improved over time but at a similar pace across sites. There was evidence of improving standards of monitoring in control and intervention sites (which started before the intervention was implemented). ${ }^{5}$ There were widespread national initiatives to improve the standard of monitoring on the wards, ${ }^{6}$ and external evidence showed increased use of hand wash materials and reduced infection rates across the whole of England over the study period. ${ }^{7} 8$ Contamination, in the sense described above, is very unlikely-controls were recruited retrospectively and data were obtained retrospectively from case notes and routine data. For these reasons, the controls would not have been aware that they were controls at the time of intervention. This is an example of an arguably unusual situation where there is specific strength in retrospective selection of control sites.

The Critical Pathway Program was an initiative started in 1993 in the Brigham and Women's Hospital (Boston, USA) to improve efficiency in service delivery for high-cost, high-volume surgical procedures. ${ }^{9}$ A controlled before-and-after evaluation for its application in colectomy, total knee replacement, and coronary artery bypass graft surgery showed substantial and statistically significant reductions in the average length of hospital stay for all three procedures in both

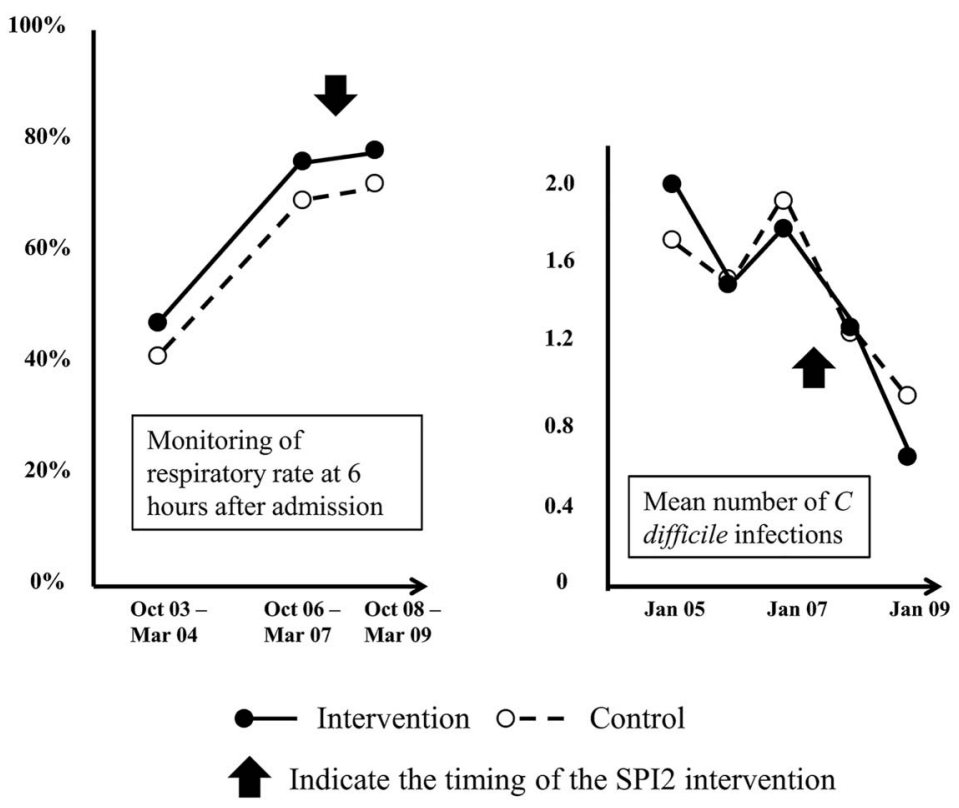

Figure 1 Key characteristics and findings of the Safer Patients Initiatives phase 2 (SPI2). This controlled before-and-after study evaluated a multi-component intervention using an organisation-wide approach to improve patient safety. The key components included interventions to facilitate generic improvement in the hospital system to reduce adverse events (such as building a good leadership to support a culture of safety as well as interventions targeting specific clinical processes that carry a relatively high risk of adverse events (such as procedures aiming to enhance infection control). Various outcomes were measured, including staff morale, culture and opinion, the quality of acute medical care and perioperative care, use of consumables for hand hygiene, adverse events and hospital mortality in older patients with acute respiratory disease, intensive care unit mortality, infection rates associated with healthcare and patients satisfaction. SPI2 was preceded by a pilot phase (SPI1) that provides data on the pre-implementation phase for certain end-points, including the two outcomes illustrated here. 

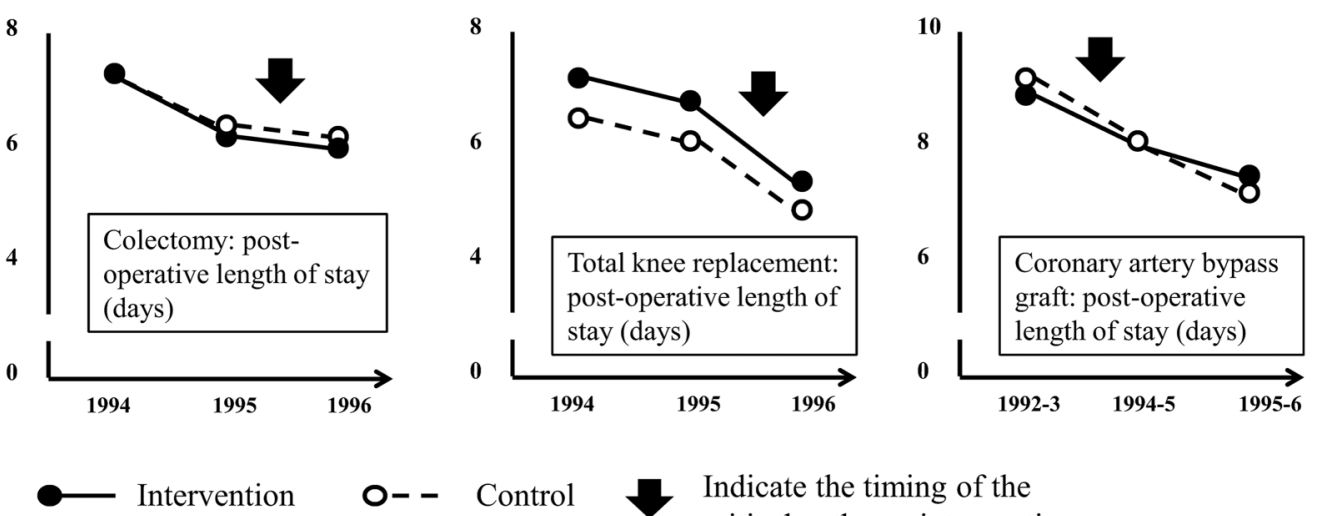

Indicate the timing of the

critical pathway intervention

Figure 2 Key characteristics and findings of the Critical Pathways Intervention. This controlled before-and-after study compared the effects of a quality improvement initiative utilising critical pathway framework on post-operative length of stay in a large teaching hospital with concurrent data from 2-3 similar neighbouring hospitals without the intervention. Multidisciplinary teams identified critical steps in the care process and specified required actions and desirable outcomes for each step. Patients entered into critical pathways were monitored and various methods of benchmarking and feedback were used in pathway management. The primary outcome was post-operative length of stay. Hospital charges and other process and clinical outcomes were also examined.

intervention and control sites. Data from the 2 years before intervention suggested that length of stay had started to decline in both intervention and control hospitals before the intervention was initiated in the former (figure 2), and external nation-wide US data showed a continuous decrease in average length of hospital admission spanning the period of the Critical Pathways Intervention, from 9.1 days in 1990 to 7.8 days in 1995 and 7.0 days in $1999 .{ }^{10}$ Staff interviews at control hospitals provided evidence that competitive pressure, rather than contamination, had triggered efforts to reduce length of stay and improve efficiency.

EQHIV was a controlled before-and-after study evaluating the effectiveness of a suite of interventions to improve the quality of care in clinics treating HIV-infected patients. ${ }^{11}$ Among the outcome measures, the proportion of patients whose viral load was adequately suppressed increased significantly within each group - by a greater extent in the intervention group (11\%; from $41 \%$ to $52 \%$ ) than the control group (6\%; from $44 \%$ to $50 \%$ ). However, the between-group difference was not statistically significant $(p=0.18)$. Compliance with a prescription guideline was already high at baseline and did not increase further in either group after the intervention (figure 3). National data from the HIV Cost and Services Utilization Study showed that EQHIV was preceded by significant improvement in care of HIV-infected adults. ${ }^{12}$ Interview of clinical directors in study sites suggested minimal contamination, as those in control sites reported many fewer quality improvement initiatives compared with intervention sites. However, attrition bias cannot be ruled out, as only $63 \%(25 / 40)$ of selected control sites provided sufficient data to be included in analysis.

MERIT was a cluster randomised controlled trial of the effectiveness of emergency teams for deteriorating non-terminal hospital patients in reducing the combined outcome of cardiac arrests without a preexisting not-for-resuscitation order, unplanned intensive care unit admissions, and unexpected deaths. ${ }^{13}$ Before the intervention began, the incidence of the outcome appeared to have already improved from 26 per 1000 admissions estimated in a previous study, ${ }^{14}$ to 6.6 and 7.1 per 1000 admissions observed at baseline for intervention and control hospitals, respectively. ${ }^{13}{ }^{15}$ Further improvement was observed in both intervention and control groups after the intervention, with no significant difference between groups

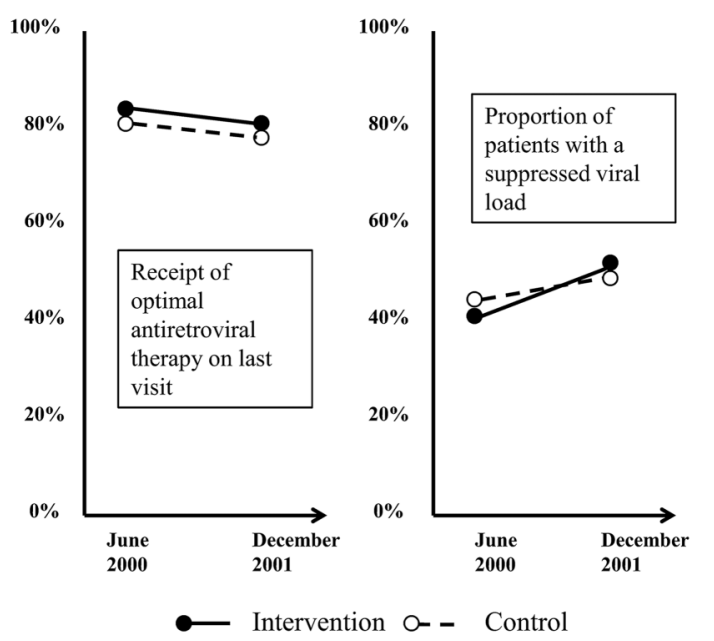

Figure 3 Key characteristics and findings of the EQHIV study. This controlled before-and-after study evaluated the effectiveness of the 'Breakthrough Series', a multi-institutional quality improvement collaborative led by Institute for Healthcare Improvement, on improving the quality of case for clinics treating HIV infected patients. The 16-month intervention involved a series of meetings (learning sessions) covering the theory and practice of quality improvement in the intervention clinics and sharing ideas and progress between them. The primary outcome measures were rates of optimal antiretroviral therapy use and control of HIV viral load. 
(reduction of 0.39 vs 1.41 per 1000 admission for intervention vs control, $\mathrm{p}=0.30$ ). Similar findings were observed for secondary outcomes (figure 4). External evidence of a secular trend and widespread adoption of medical emergency teams comes from a national registry in which about $30 \%$ of all intensive care units provided relevant data. ${ }^{16}$ The risk of contamination was minimised by agreement of control hospitals not to publicise the intervention internally and not to change the operation of their cardiac arrest team during the study period.

In summary, there is evidence for a secular trend in all four cases. The case for a rising tide is strongest for SPI2 and MERIT where data from both within and outside the study pointing towards a system-wide secular trend and evidence for alternative explanations can largely be ruled out. For the remaining cases, there is some uncertainty, mainly arising from lack of evidence to eliminate alternative explanations. On the whole, the evidence (summarised in table 1) indicates that a secular trend is likely to have contributed to the null results observed in all four studies.

\section{DISCUSSION}

\section{What causes a rising tide?}

Widespread concern about an issue such as hospital-acquired infection or medication error may motivate multiple changes throughout a system that includes, but is not limited to, sites involved in a research study. Exactly how these changes are propagated is a large subject for study, save to say that

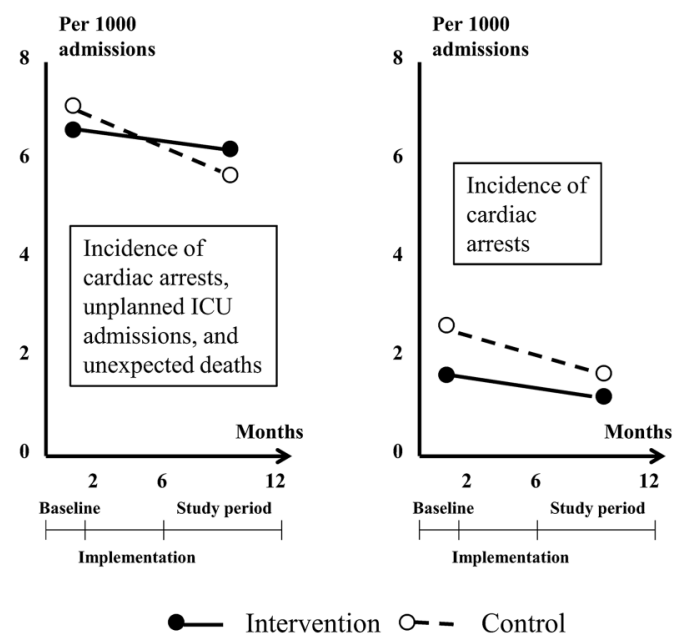

Figure 4 Key characteristics and findings of the MERIT study. This cluster randomised controlled trial (RCT) investigated the effectiveness of introducing a medical emergency team (MET) that could be summoned when non-terminal hospital patients showed signs of physiological instability and deterioration. Staff in the intervention hospitals were trained over 4-months to identify and respond to patients requiring the attention of the team. The primary outcome was the combined incidence of cardiac arrests without a pre-existing not-for-resuscitation order. unplanned intensive care unit admissions and unexpected deaths measured before and after implementation. human behaviour is strongly influenced by prevailing social attitudes and practice. ${ }^{17}$ Two points can be made about the phenomenon of the spread of behaviour in a community of practitioners:

1. It is not necessary to postulate that the way in which organisations respond to social 'forces' is the same everywhere. Services may be improved in a number of separate ways, ${ }^{18}$ and improvement across the system might arise from intervention and non-intervention sites adopting the same practices, separate practices of similar efficiency, or a mixture of similar and different practices. An analogy of the multifarious ways that social forces may cause a rising tide is shown in box 1 . The intervention group is also subject to the rising tide. The measured intervention effect in a study inclines towards the null if the effect of the intervention attenuates with increasing 'dose' and/or if the headroom for further improvement is consumed.

2. A rising tide can only produce a null result if there is at least some temporal overlap between widespread promulgation of the interventions and the evaluation of a particular intervention. However, it is not necessary for system-wide change and research to start simultaneously and such timing is unlikely given the lag in establishing research projects. Indeed improvement originated before the study got underway in three of the four above examples (table 1).

\section{Detecting a rising tide explanation}

A rising tide phenomenon is, in essence, a pronounced secular trend created by social responses to a particular issue which has gained widespread attention. While it is impossible to find incontrovertible proof of a rising tide explanation, we have assembled a set of criteria that should be taken into account in the interpretation of controlled evaluations that have generated a null result associated with similar improvement in both intervention and control groups

\section{Box 1 Evolutionary analogy of the rising tide phenomenon}

A naturalist studying desert fauna may notice that they have certain features in common. It might first be observed that mice are sand-coloured. Then that the snakes, lizards and small birds are all of similar hue. The naturalist may observe that this would increase their efficiency as both prey and/or predator (both, in the case of the snake). Under the same external influence (changes in physical environment), organisms evolve similar outcomes (a sandy colour) by different means (distinct biochemical pathways). So it is that, under the same primary driver (changes in the social environment), organisations evolve similar outcomes (fewer infections) by different means (such as promoting hygienic practices and screening all patients for resistant bacteria on admission). 
(table 1). Indeed, possible influence of a secular trend was mentioned or alluded to by the authors of all the case examples we presented here. It must be emphasised that a rising tide does not preclude a positive result. The intervention may augment widespread contemporaneous change because the intervention is different (at least in part), and/or administered with different intensity. For instance, in a controlled evaluation of a team training programme for operating room personnel, a statistically significant reduction in risk-adjusted surgical mortality rate was observed, despite a $7 \%$ decrease in annual mortality rate in the control group. ${ }^{19} \mathrm{~A}$ tide may also recede, in which case a successful intervention may be one that arrests decline, but this would be manifested as a positive result, not a null result.

\section{Rising tide in applied health research}

Situations analogous to the rising tide phenomenon can occur in a variety of applied health research-for example, in a trial of screening for prostate cancer, where a substantial proportion of the population (and hence the control group) underwent screening. ${ }^{20}$ Likewise, an educational package for general practitioners to apply more intensive antidepressant treatment was evaluated at a time when the idea was already getting national publicity. ${ }^{21}$ Many other examples can be found in the realm of service delivery interventions. ${ }^{22}{ }^{23}$ Most recently, the rising tide phenomenon is likely to have contributed to the null findings from two independent analyses of the effect of participation in the American College of Surgeons National Surgical Quality Improvement Program (ACS NSQIP), where mortality and certain other outcomes improved in both intervention and control groups, ${ }^{24} 25$ and in the English Matching Michigan study where the rate of decline in central venous catheter bloodstream infection following the introduction of the intervention in intensive care units was not significantly different from a concurrent temporal trend. ${ }^{23}$

\section{Does it matter?}

We have described a set of criteria to help decide whether a null result in the face of improving outcomes can be attributed to a rising tide (table 1). One subset of criteria concerns a convincing alternative explanation, particularly contamination. It could be argued that a null result needs no further explanation once one is satisfied that it has been measured with sufficient precision and decided that an alternative explanation, such as contamination, can be excluded. Contrary arguments are now given based on two rather distinct philosophical traditions.

1. We draw attention to a distinction made by Schwartz and Lellouch ${ }^{26}$ between pragmatic and explanatory motivations for a study. The former consists of generating information to inform a particular prespecified decision, and the second consists of generating an understanding of causal mechanisms. A null result in the face of a rising tide fulfils the first, but not the second, requirement. It fulfils the first (pragmatic) requirement because, if a study designed (and powered) around the decision makers' requirements is assumed, an incremental effect size sufficient to justify the marginal costs of the intervention is excluded. However, the second (explanatory) requirement is unsatisfied, since it does not indicate what the effect of the study intervention would be in a system that was not experiencing a positive temporal trend. In such a system, the intervention would not be 'competing' with other positive changes in the system.

2. The second philosophical argument turns on the idea that it is wrong to make decisions based solely on a statistical convention, ${ }^{27}$ as pointed out in Sir Bradford Hill's famous lecture. ${ }^{1}$ To put this another way, data should contribute to an understanding of causal mechanisms (theory), and the rising tide may help explain why an intervention that was expected to prove effective yielded a null result.

\section{Recommendations for future practice}

Having discussed the idea of a secular trend phenomenon, we propose here some options that can be considered alongside established guidelines ${ }^{28-31}$ during the design of evaluation studies for service and policy interventions in order to facilitate correct interpretation of study findings.

1. In many cases, at least some of the study end points will be available from routine administrative databases or independent surveys regularly carried out nationally. This will allow verification of whether a change observed in the evaluation study is associated with the study participation itself or is similarly observed elsewhere outside the study, thereby providing strong evidence of a secular trend, at least as far as shared end points are concerned. This was the case in the SPI2 study.

2. Qualitative data may provide evidence to explain study results; $;^{5} 32$ in the case of SPI2, behaviour change was driven by factors in the external environment in both intervention and control sites.

3. Obtaining multiple measurements spanning the pre- and post-intervention period - that is, a controlled interrupted time series. ${ }^{29}$ Multiple observations before the intervention phase may provide evidence of long-term secular trends in both control and intervention groups. 3334

4. Prior to the start of data collection, the sample size can be adjusted to take account of secular trends when these are expected. Such analysis can be used to assess the feasibility and value of an evaluation study before it is commissioned, or to inform a decision on whether to extend an ongoing study by increasing its size or to terminate it on grounds of 'futility'. 3536

5. Considering designs that allow temporal effects to be modelled. One example is a step wedge design, ${ }^{37}$ which uses randomisation as a method to determine the order in which centres on a waiting list receive the 
intervention. It has many logistical, political and even ethical advantages over a parallel design, ${ }^{28} 38 \quad 39$ and (given a sufficiently large sample) also allows the intervention effect, general temporal effects, and any effect on the intervention at the time it was introduced to be modelled.

\section{CONCLUSION}

Social pressure that triggers the development and evaluation of a service delivery intervention may at the same time drive spontaneous, widespread changes in a health system leading to improvement across the board, which we describe here as a rising tide. Controlled evaluation studies undertaken amidst a rising tide may yield a null result because incremental effects are similar between intervention and nonintervention sites. Recognition of a rising tide is important because, while the null result demonstrates pragmatically that the intervention does not produce sufficient incremental benefit in this particular scenario, it leaves open the possibility that the intervention could work in a different scenario where a rising tide is absent.

In this paper we offer four case studies of evaluations of complex interventions to illustrate a rising tide phenomenon, and suggest a framework to assess evidence either supporting or refuting its presence. Our aim is to raise awareness of the phenomenon and of its potential implications in the design and interpretation of evaluation studies. Further work to gather empirical evidence on the occurrence of such a phenomenon and to develop methods to delineate its impact from other bias is the next step. This in turn will provide guidance for health services researchers and decision makers on the optimal actions to take in the face of a rising tide.

Contributors RJL conceived the idea for the paper, wrote the first draft, led the writing of the paper and is the guarantor. Y-FC compiled the case examples and helped to draft the paper. $\mathrm{KH}$ and AJS read and commented on drafts and provided critical insight into the development of the paper.

Funding RJL and Y-FC have received funding from the UK National Institute for Health Research (NIHR) through the Collaboration for Leadership in Applied Health Research and Care West Midlands (CLAHRC WM) programme. RJL and KH had financial support from the Medical Research Council (MRC) Midland Hub for Trials Methodology Research (grant No G0800808).

Disclaimer The views expressed here are those of the authors and not necessarily those of the NHS, the NIHR or the Department of Health.

Competing interests RJL was the principal investigator for the evaluation study of SPI2 described in this paper.

Provenance and peer review Not commissioned; externally peer reviewed.

Open Access This is an Open Access article distributed in accordance with the terms of the Creative Commons Attribution (CC BY 4.0) license, which permits others to distribute, remix, adapt and build upon this work, for commercial use, provided the original work is properly cited. See: http://creativecommons.org/licenses/by/4.0/

\section{REFERENCES}

1 Hill AB. The environment and disease: association or causation? Proc R Soc Med 1965;58:295-300.

2 Keogh-Brown MR, Bachmann MO, Shepstone L, et al. Contamination in trials of educational interventions. Health Technol Assess 2007;11:1-107.

3 Torgerson DJ. Contamination in trials: is cluster randomisation the answer? BMJ 2001;322:355-7.

4 Benning A, Dixon-Woods M, Nwulu U, et al. Multiple component patient safety intervention in English hospitals: controlled evaluation of second phase. BMJ 2011;342:d199.

5 Benning A, Ghaleb M, Suokas A, et al. Large scale organisational intervention to improve patient safety in four UK hospitals: mixed method evaluation. BMJ 2011;342:d195.

6 National Institute for Health \& Clinical Excellence. Acutely ill patients in hospital. Recognition of and response to acute illness in adults in hospital. NICE clinical guideline 50, 2007.

7 Health Protection Agency. Surveillance of healthcare associated infections report: 2008. 2008. http://www.hpa.org.uk/webc/ HPAwebFile/HPAweb_C/1216193833496 (accessed 21 Nov 2011).

8 Stone S, Slade R, Fuller C, et al. Early communication: Does a national campaign to improve hand hygiene in the NHS work? Initial English and Welsh experience from the NOSEC study (National Observational Study to Evaluate the CleanYourHandsCampaign). J Hosp Infect 2007;66:293-6.

9 Pearson SD, Kleefield SF, Soukop JR, et al. Critical pathways intervention to reduce length of hospital stay. Am J Med 2001;110:175-80.

10 National Center for Health Statistics. Health, United States, 2004. With Chartbook on Trends in the Health of Americans. 2004. http://www.ncbi.nlm.nih.gov/books/NBK20751/pdf/ TOC.pdf (accessed 2 Feb 2015).

11 Landon BE, Wilson IB, McInnes K, et al. Effects of a quality improvement collaborative on the outcome of care of patients with HIV Infection: The EQHIV Study. Ann Intern Med 2004;140:887-96.

12 Shapiro MF, Morton SC, McCaffrey DF, et al. Variations in the care of HIV-infected adults in the United States. JAMA 1999;281:2305-15.

13 Hillman K, Chen J, Cretikos M, et al., MERIT Study Investigators. Introduction of the medical emergency team (MET) system: a cluster-randomised controlled trial. Lancet 2005;365:2091-7.

14 Bristow PJ, Hilman KM, Chey T, et al. Rates of in-hospital arrests, deaths and intensive care admissions: the effect of a medical emergency team. Med J Aust 2000;173:236-40.

15 Cretikos MA, Chen J, Hilman KM, et al. The effectiveness of implementation of the medical emergency team (MET) system and factors associated with use during the MERIT study. Crit Care Resusc 2007;9:205-12.

16 Jones D, George C, Hart G, et al. Introduction of medical emergency teams in Australia and New Zealand: a multi-centre study. Crit Care 2008;12:R46.

17 Bandura A, Grusec JE, Menlove FL. Some social determinants of self-monitoring reinforcement systems. J Pers Soc Psychol 1967;5:449-55.

18 Sheldon T. Safety alerts on drugs-how trusts follow the rules. J Health Serv Res Policy 2010;15(Suppl 1):44-7.

19 Neily J, Mills PD, Young-Xu Y, et al. Association between implementation of a medical team training program and surgical mortality. JAMA 2010;304:1693-700. 
20 Andriole GL, Grubb RL, Buys SS, et al. Mortality results from a randomized prostate-cancer screening trial. $N$ Engl J Med 2009;360:1310-19.

21 Thompson C, Kinmonth AL, Stevens L, et al. Effects of a clinical-practice guideline and practice-based education on detection and outcome of depression in primary care: Hampshire Depression Project randomised controlled trial. Lancet 2000;355:185-91.

22 Solberg LI, Kottke TE, Brekke ML, et al. Failure of a continuous quality improvement intervention to increase the delivery of preventive services: a randomized trial. Eff Clin Pract 2000;3:105-15.

23 Bion J, Richardson A, Hibbert P, et al. 'Matching Michigan': a 2-year stepped interventional programme to minimise central venous catheter-blood stream infections in intensive care units in England. BMJ Qual Saf 2013;22:110-23.

24 Osborne NH, Nicholas LH, Ryan AM, et al. Association of hospital participation in a quality reporting program with surgical outcomes and expenditures for Medicare beneficiaries. JAMA 2015;313:496-504.

25 Etzioni DA, Wasif N, Dueck AC, et al. Association of hospital participation in a surgical outcomes monitoring program with inpatient complications and mortality. JAMA 2015;313:505-11.

26 Schwartz D, Lellouch J. Explanatory and pragmatic attitudes in therapeutical trials. J Chronic Dis 1967;20:637-48.

27 Lilford RJ, Braunholtz D. For Debate: The statistical basis of public policy: a paradigm shift is overdue. $B M J$ 1996;313:603-7.

28 Brown C, Hofer T, Johal A, et al. An epistemology of patient safety research: a framework for study design and interpretation. Part 2. Study design. Qual Saf Health Care 2008;17:163-9.

29 Craig P, Cooper C, Gunnell D, et al. Using natural experiments to evaluate population health interventions: guidance for producers and users of evidence. 2011. http://www.mrc.ac.uk/ Utilities/Documentrecord/index.htm?d=MRC008043 (accessed 4 Apr 2012).

30 Craig P, Dieppe P, Macintyre S, et al. Developing and evaluating complex interventions: the new Medical Research Council guidance. BMJ 2008;337:a1655.

31 Eccles M, Grimshaw J, Campbell M, et al. Research designs for studies evaluating the effectiveness of change and improvement strategies. Qual Saf Health Care 2003;12:47-52.

32 Lilford RJ, Chilton PJ, Hemming K, et al. Evaluating policy and service interventions: framework to guide selection and interpretation of study end points. BMJ 2010;341:c4413.

33 Cochrane Effective Practice and Organisation of Care Group. Study designs accepted in EPOC reviews. 2013. http://epoc. cochrane.org/epoc-resources (accessed 17 May 2013).

34 Shadish WR, Cook TD, Campbell DT. Experimental and quasi-experimental designs for generalized causal inference. Boston: Houghton Mifflin, 2002.

35 Hemming K, Girling AJ, Sitch AJ, et al. Sample size calculations for cluster randomised controlled trials with a fixed number of clusters. BMC Med Res Methodol 2011;11:102.

36 Lilford R, Jecock R, Shaw $\mathrm{H}$, et al. Commissioning health services research: an iterative method. J Health Serv Res Policy 1999;4:164-7.

37 Hemming K, Haines TP, Chilton PJ, et al. The stepped wedge cluster randomised trial: rationale, design, analysis, and reporting. BMJ 2015;350:h391.

38 Brown CA, Lilford RJ. The stepped wedge trial design: a systematic review. BMC Med Res Methodol 2006;6:54.

39 Mdege ND, Man MS, Taylor CA, et al. Systematic review of stepped wedge cluster randomized trials shows that design is particularly used to evaluate interventions during routine implementation. J Clin Epidemiol 2011;64:936-48. 